\title{
Using a Combination of Matrix Approach to Robustness Analysis (MARA) and Fuzzy DEMATEL-Based ANP (FDANP) to Choose the Best Decision
}

\author{
Ali Sorourkhah \\ Department of Management, \\ Ayandegan Institute of Higher Education, Tonekabon, Iran. \\ E-mail: ali.sorourkhah@gmail.com, ali.sorourkhah@semnan.ac.ir \\ S. A. Edalatpanah \\ Department of Applied Mathematics, \\ Ayandegan Institute of Higher Education, Tonekabon, Iran. \\ Corresponding author: saedalatpanah@gmail.com, s.a.edalatpanah@aihe.ac.ir
}

(Received on June 10, 2021; Accepted on December 19, 2021)

\begin{abstract}
In today's world, selecting a suitable, reasonable option is challenging for any decision-maker. Where does this problem arise from? According to the decision-making literature, complexity "multiple criteria and interrelationship among them" and uncertainty "the vagueness of judgments and future uncertainty" have made decision-making one of the most significant problems for people. Though multiple-criteria decision-making (MCDM) methods can help decision-makers select the best alternative among multiple criteria and consider their interrelationships, they do not deal with uncertainty. Fuzzy set theory handles uncertainty arising from the vagueness of human thoughts and language in making decisions. Still, the MCDM or FMCDM techniques fail to formulate the probable futures since they use the present information and judgments to collect the data, while in the real world, choosing a method that can deal with the uncertain environment and reduce its impact on outcomes will be effective. Moreover, soft OR methods, such as robustness analysis, strategic choice approach, etc., can deal with future uncertainty but not complexity. Thus, we will use a combination of the Matrix Approach to Robustness Analysis (MARA) and Fuzzy DEMATEL-based ANP (FDANP) to address the weakness mentioned above. This hybrid method has two significant advantages: it can (i) consider numerous scenarios, criteria, and alternatives as well as the interdependency among criteria to address the complexity aspect, and (ii) examine the option performance among the different possible futures and the fuzzy nature of the problem owner's judgments to address the uncertainty aspect. This method is applied to a case related to starting a new business in Iran. The results show that by concerning the environmental situations and the possible future of Iran, education service is the most robust business to start.
\end{abstract}

Keywords- Decision-making, Robustness analysis, Complexity and uncertainty, Fuzzy DEMATEL-based ANP (FDANP).

\section{Introduction}

For so many years, decision-making has been a regular, ongoing thing (task), but nowadays, it is challenging (Lin and Pham, 2019). No matter what we do, whether a manager, a mother, or even a jobless person, everybody has to make different decisions during the day. This process forces us to select between various options. It is said that choosing a suitable choice is one of the most significant problems for decision-makers (Nakhjavani et al., 2014). Where does this problem arise from? Reviewing decision-making literature addresses two critical factors: complexity and uncertainty (Ocampo et al., 2019). Complexity includes two main aspects: (A) identifying effective criteria (Taati and Dooki, 2017) in the situation in which numerous variables are affecting the consequences of the decision and (B) determining the relationships among the evaluation criteria 
(Salimi and Edalatpanah, 2020). Uncertainty includes two main aspects as well: (A) the uncertainty of individual judgment (Ocampo et al., 2015) and (B) future uncertainty - the future which will most probably be different from what we have expected (Rudd et al., 2008). The last aspect of the uncertainty is related to the environmental factor and their changes affecting the decision outcome.

Managing complexity needs to grasp the interrelationships among interacting criteria affecting the situation (Kim et al., 2021). The diversity of criteria and various dependencies among them have caused selecting the best option to be very complicated (Arjomandi et al., 2021). While The effectiveness of decision-making depends mainly on DMs ability to analyze these interrelationships (Lin and $\mathrm{Wu}, 2008$ ), the number of criteria and the interactions among them make the interdependency more complex (Song et al., 2020). In such situations, applying multi-criteria decision-making (MCDM) techniques seems reasonable (Taati and Dooki, 2017; Avikal et al., 2020; Kharisma and Ardi, 2020). MCDM is continuously growing because of its ability to capture multidimensionality (Kavta and Goswami, 2021). Among all its well-known methods, one of the most widely used MCDM is the Analytic Network Process (ANP), a mathematical theory that can systematically overcome all types of dependencies (Hsu et al., 2012). Applying the ANP method will be advisable when the problem elements form a network structure (Tadić et al., 2014). Another helpful method developed to make decisions in a complex environment (Dinçer et al., 2019) is the Decision-Making Trial and Evaluation Laboratory, DEMATEL (Liou et al., 2018). DEMATEL makes a structural model and then analyzes the influence relation among multiple criteria (Wu and Lee, 2007; Chang et al., 2011). More recently, calculating the criteria weight by using a combination of DEMATEL and ANP (Hsu et al., 2012; Sudarmin and Ardi, 2020), DANP, has been addressed by so many researchers (Huang, 2012; Vinodh and Swarnakar, 2015; Busyra and Ardi, 2020). Applying DANP, decision-makers can determine the key factors and their weights (Hu et al., 2014). Though the ANP, DEMATEL, and DANP techniques solve the complexity aspect of the problem, none of them considers neither future uncertainty nor individual judgment uncertainty.

There is no gainsaying that a lot of referable studies have been conducted to respond to one aspect of uncertainty by using fuzzy set theory (Sagnak et al., 2020; Akram et al., 2021). Fuzzy logic is an approach to calculating the values based on "degrees of truth" instead of the usual Boolean "true or false" logic (Edalatpanah, 2020). Because human judgment about preferences is often unclear and hard to estimate by exact numerical values, it is constructive to deal with the vagueness of human thoughts and language in making decisions (Lin and Wu, 2008; Huang and Ting, 2012). The integration of fuzzy set theory with MCDM methods has been reported vastly in the literature (Ocampo et al., 2020) and is not enumerated here for brevity. However, while decision-making problems need to be carried out under uncertainty (Seker and Zavadskas, 2017), another aspect of that, future uncertainty, is neglected in the MCDM literature. MCDM and FMCDM models can deal with both qualitative and quantitative data; can consider a sufficient number of criteria, options, etc.; can formulate the relationships among different criteria; and, if using fuzzy set theory, can model the vagueness of verbal judgments. Nevertheless, none analyze the consequences of decisions (alternatives, options, etc.) and the environmental factors changing the current situation. Imagine you want to choose a supplier between $S_{1}$ and $S_{2}$ while you live in Iran, and the quality of raw material is your only criterion. $S_{1}$ located in your country has low-quality and $S_{2}$ located in Japan has high-quality. After a few months, while you choose $S_{2}$, this supplier refuses to supply you because of the Joint Comprehensive Plan of Action (JCPOA). What happens now? Is still your decision suitable and reasonable? It indicates while we live in a turbulent environment, we have to consider the possible scenarios of the future. 
There are a few approaches in which the future scenarios form the basis of the analysis. Scenario Planning (SP) is a well-known approach to support strategic planning and decision-making (Greiner et al., 2014). It defines a specific set of uncertainties, what the future is going to be and how the environment will change over time in light of that future; Strategic Choice Approach (SCA) deals with the uncertainty by introducing three different categories: Uncertainties about our working environment (UE), Uncertainties about our guiding values (UV), and Uncertainties about choices on related agendas (UR); Robustness Analysis (RA) is an approach to assess the primary sequential decisions in the sense that robustness and debility of choice in the future alternatives is examined to make a decision that could provide acceptable and satisfactory results among more futures and simultaneously followed by fewer adverse outcomes (Rosenhead, 1980, 2011). Having some distinct advantages in considering uncertainty, none of these approaches can deal with both aspects of complexity. Specifically, scenario planning models often include only three, four, or five scenarios and can not deal with various criteria affecting the problem to address the first aspect of the complexity. The strategic choice approach is a reliable one to handle the uncertainty surrounding the decision-making problem. However, it cannot model the relationships among the criteria and their possible future situations. And robustness analysis, which is able to handle one aspect of the complexity, the number of the criteria, scenarios, etc., does not consider the criteria interrelationships. Hence, we believe that applying a combinational approach addressing all the above constraints is essential.

As applying an inappropriate approach in the decision-making process leads to inaccurate results (Dinçer et al., 2019), choosing an approach that can deal with the uncertain environment and reduce its impact on outcomes (Chutia and Gogoi, 2018), and that covers complexity aspects will be effective. This situation forces us to combine some suitable approaches because any of the above ones consider one or two aspects of either complexity or uncertainty. The MCDM approaches fail to formulate the probable futures since decision-makers tend to give assessments according to their past experiences and knowledge (Lin and $\mathrm{Wu}, 2008$ ), while they must consider the changing conditions of today's environment (Azar and Sorourkhah, 2015). Due to human capacity constraints, scenario Planning cannot deal with complexity (Månsson, 2016). Robustness Analysis, which is more uncomplicated and more accessible than SCA to understand for the managers and participants (Namen et al., 2010), has some significant weaknesses (Sorourkhah and Edalatpanah, 2021). It considers a small number of scenarios, is unable to deal with numerous criteria, and above all, fails to determine the results of some decisions in future scenarios. It should be said that the Matrix Approach to Robustness Analysis MARA (Sorourkhah et al., 2018, 2019) covers all the RA weaknesses but the relationships among the criteria. So, we will use a combination of the Matrix Approach to Robustness Analysis, MARA, and Fuzzy DEMATEL-based ANP, DANP, to address the weakness mentioned above. "MARA can deal with one aspect of complexity (numerous criteria, options, scenarios, etc.) and one aspect of uncertainty (future uncertainty), and FDANP can deal with another aspect of complexity (criteria interdependency) and another aspect of uncertainty (uncertainty of individual judgment). So, the proposed approach considers the dimensions of both complexity and uncertainty". Robustness analysis, in fact, is an appropriate model to address future uncertainty because it analyzes options (decisions) according to the future situation of different criteria. The matrix approach can deal with a large number of criteria, scenarios and options at an appropriate time. And fuzzy DANP can handle the criteria interdependency and judgment vagueness. The proposed approach can be helpful in complex and uncertain environments where finding, analyzing and choosing the best option is challenging. 
The proposed approach will be applied to a problem related to a decision-maker who wants to start a business in Ramsar, Iran. This case is an MCDM one with multiple elements (criteria, scenarios, etc.), interdependency among criteria, future uncertainty, vagueness of human judgments, and some options to be selected. In this regard, our paper is organized in the following arrangements. In Section 2, our methodology, including the mixture of MARA and FDANP, is described in detail. In Section 3, the methodology is applied to a case study, and the results are presented and compared with the previous study. Finally, conclusions are drawn in Section 4.

\section{Methodology}

To select the best option, we combine the MARA approach with FDANP, the process of which is illustrated in Figure 1. Accordingly, we need first to identify the main options, $M O_{i}, i=1, \ldots, r$, implying the result of choosing several predefined options $O_{j}, j=1, \ldots, m$, as follows:

$\mathrm{MO}_{\mathrm{i}}=\mathrm{O}_{\mathrm{j}_{1}} \mathrm{O}_{\mathrm{j}_{2}} \ldots \mathrm{O}_{\mathrm{j}_{\mathrm{i}}} \mathrm{i}=1, \ldots, \mathrm{r}$,

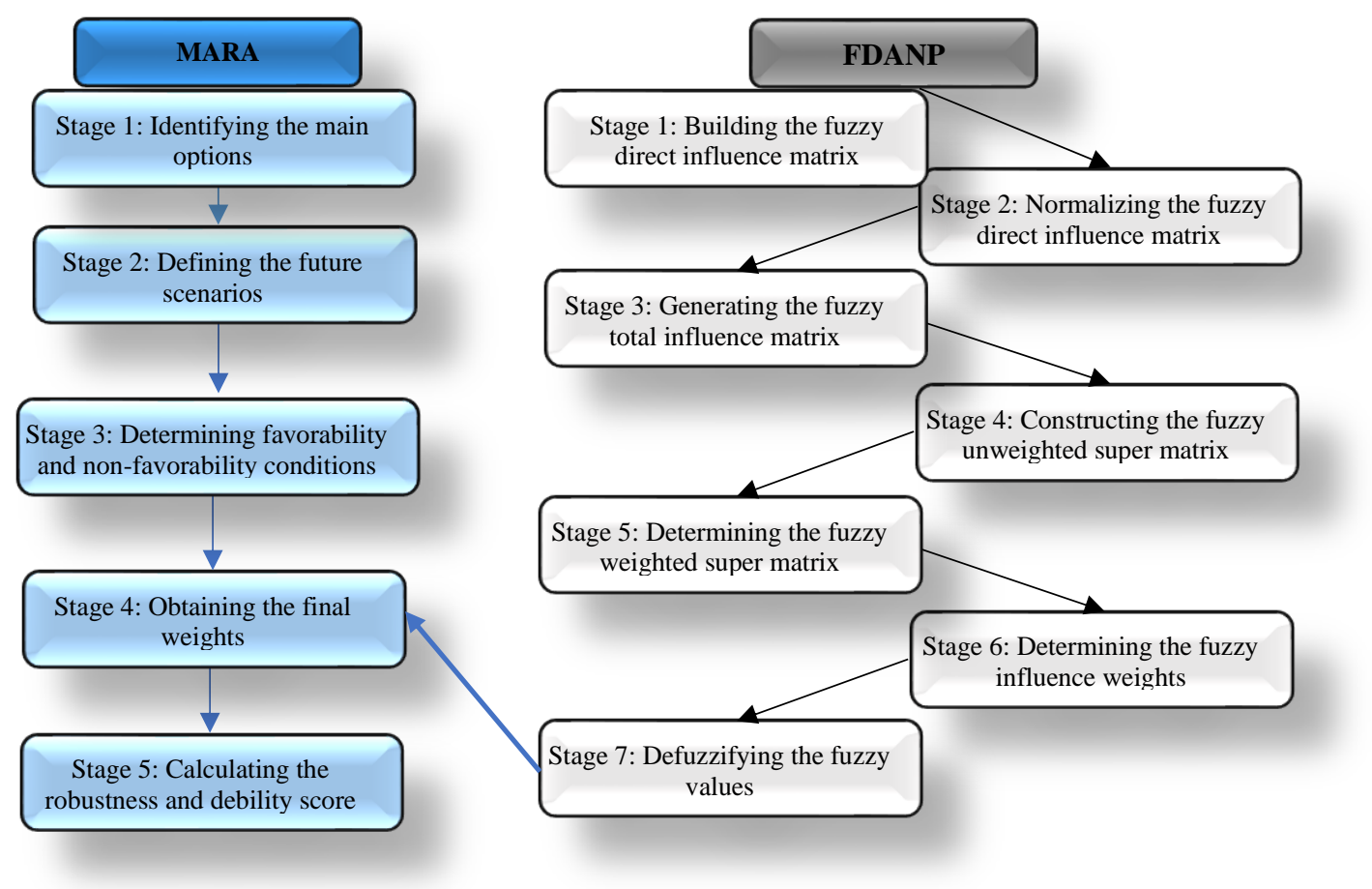

Figure 1. The proposed model flowchart.

In the next step, we need to define the scenario matrix $S$. The probable situations (states) of the criteria (indicators) determined by decision-makers will shape this matrix. The columns of the matrix $S\left(S c_{i}\right)$ show the number of scenarios, and its rows indicate a given situation of each indicator, $S=\left[S c_{1}, S c_{2}, \ldots, S c_{q}\right]$

In the third step, for option $O j$, decision-makers specify favorability $\left(\mathrm{Oj}^{+}\right)$and non-favorability $\left(\mathrm{Oj}^{-}\right)$ conditions based on the different situations of the indicators displayed in the previous step. Afterward, the condition matrix $C$ of the order $\mathrm{n} \times 2 \mathrm{~m}$ can be defined as follows:

$C=\left[O_{1}{ }^{+}, \mathrm{O}_{1}{ }^{-}, O_{2}{ }^{+}, O_{2}{ }^{-}, \ldots, O_{m}{ }^{+}, O_{m}{ }^{-}\right]$. 
Before the next step, we apply FDANP to gain the weights of environmental indicators and consider their interdependency as follows:

Step 1. Building the fuzzy direct influence matrix $(\tilde{A})$ with respect to Table 1 . For the sake of brevity, we just introduce FDANP steps. For more details, if necessary, please see (Hamedi and Mehdiabadi, 2020; Karuppiah et al., 2020; Kim et al., 2021).

Table 1. Verbal variables and the TFNs (Kim et al., 2021).

\begin{tabular}{|c|c|c|c|c|c|}
\hline Verbal variables (influence) & No & Low & Medium & High & Very high \\
\hline Fuzzy numbers & $(0.0,0.0,0.25)$ & $(0.0,0.25,0.5)$ & $(0.25,0.5,0.75)$ & $(0.5,0.75,1.0)$ & $(0.75,1.0,1.0)$ \\
\hline
\end{tabular}

Step 2. Deriving $(\widetilde{N})$ through normalizing $(\tilde{A})$.

Step 3. Generating the fuzzy total influence matrix $(\tilde{T})$ defined as:

$\tilde{T}=\widetilde{N}(\tilde{I}-\widetilde{N})^{-1}$

Step 4. Constructing the fuzzy unweighted supermatrix $\left(\widetilde{W}_{c}\right)$.

The fuzzy total influence relation matrix of criteria is expressed by $\left(\widetilde{T}_{c}\right)$ in Eqn. (5):

$\tilde{T}_{c}=\left(T_{c}^{l}, T_{c}^{m}, T_{c}^{h}\right)$.

The normalized influence matrix for criteria assigned by $\left(\widetilde{T}_{c}^{\alpha}\right)$ in Eqn. (6):

$\tilde{T}_{c}^{\alpha}=\left(T_{c}^{\alpha l}, T_{c}^{\alpha m}, T_{c}^{\alpha h}\right)$.

Taking the transposition of normalized submatrixes, we will construct $\widetilde{W}_{c}$ in Eqn. (7):

$\widetilde{W}_{c}=\left(T_{c}^{\alpha}\right)^{\prime}=\left(W^{l}, W^{m}, W^{h}\right)$.

Step 5. Determining the fuzzy weighted supermatrix $(\widetilde{W})$.

Normalizing the fuzzy total influence relation matrix of clusters, $\left(\widetilde{T}_{D}\right)$, we will obtain $\left(\widetilde{T}_{D}^{\alpha}\right)$. Then, multiplying $\left(\widetilde{T}_{D}^{\alpha}\right)$ and $\widetilde{W}_{c}$, we will calculate the fuzzy weighted supermatrix $(\widetilde{W})$ in Eqn. (8):

$\widetilde{W}^{\alpha}=\widetilde{T}_{D}^{\alpha} \otimes \widetilde{W}_{c}$

Step 6. Determining the fuzzy influence weights $\left(\widetilde{W}^{*}\right)$.

Reaching a stable supermatrix, $\widetilde{W}^{\alpha}$ can be raised to limiting powers in Eqn. (9):

$\lim _{z \rightarrow \infty}\left(\widetilde{W}^{\alpha}\right)^{z}$.

Step 7. Defuzzifying the fuzzy values.

The Best Non-fuzzy Performance (BNP) defuzzification method is used in defuzzifying the fuzzy values into crisp values (Chen et al., 2008). The BNP of a triangular fuzzy number a $=(1, \mathrm{~m}, \mathrm{u}) \mathrm{can}$ be expressed as:

$B N P=l+\frac{(u-l)+(m-l)}{3}$

Now, in the last step of the MARA approach, we can define the robustness-debility matrix $(Z)$. The columns of matrix $Z$ corresponds to different scenarios $\left(S c_{i}\right)$, and its rows correspond to alternative 
options $(\mathrm{O} j)$. To determine the element $(j, i)$ of matrix $Z$, we have to compare each scenario with the favorability $\left(\mathrm{Oj}^{+}\right)$and non-favorability $\left(\mathrm{Oj}^{-}\right)$condition matrix $C$. Accordingly, we will consider a positive score (equal to the indicator weight) for each compliancy of $S c_{i}$ with $O_{j}^{+}$and a negative score (equal to the indicator weight) for each compliancy of $S c_{i}$, with $O_{j}^{-}$. Sum of these scores will be the element $Z_{j i}$.

Next, using Eqn. (11), we can calculate the robustness $(R)$ scores of the options (Rosenhead, 1980): $R_{j}=\frac{n(r)}{q}$,

in which $n(r)$ indicates the number of positive elements of the $j$ th row of the matrix $Z$ and $q$ shows the total number of the elements of the $j$ th row. For the debility $(D)$ scores, we have:

$D_{j}=\frac{n(d)}{q}$,

in which $n(d)$ indicates the number of negative elements of the $j$ th row of matrix $Z$.

Finally, for each main option $M O_{i}$, the sum of the elements of $R$ corresponding to the predefined options (see Eqn. (1)) determines the robustness level, and the debility level will be calculated in the same way. The best option has the highest robustness score.

\section{Case Study}

This study is related to a decision-maker who wanted to start a viable business in Ramsar, Iran. Because this problem has an MCDM structure, we applied the proposed method to solve the problem. Initially, she considered two main options: product business $\left(M O_{1}\right)$, including a tea manufacturing facility $\left(O_{1}\right)$ or producing artisanal handicrafts $\left(O_{2}\right)$, and service business $\left(M O_{2}\right)$, including cafeteria $\left(\mathrm{O}_{3}\right)$, tourism $\left(\mathrm{O}_{4}\right)$, or education service $\left(\mathrm{O}_{5}\right)$. In step 1, we identified the main options based on the Eqn. (1) as follows:

$$
\begin{aligned}
& M O_{1}=O_{1} \oplus O_{2}, \\
& M O_{2}=O_{3} \oplus O_{4} \oplus O_{5} .
\end{aligned}
$$

In step 2, we defined future scenarios according to different situations shown in Table 2.

Table 2. Factors and different states of them.

\begin{tabular}{|l|l|l|}
\hline Factors & Indicators & Situations \\
\hline \multirow{2}{*}{ Economic } & Currency exchange rate & Decrease $\left(f_{11}\right)$, Increase $\left(f_{12}\right)$ \\
\cline { 2 - 3 } & Inflation rate & Less than $\% 20\left(f_{21}\right)$, More than $\% 20\left(f_{22}\right)$ \\
\hline \multirow{2}{*}{ Market } & Competition level & Decrease $\left(f_{31}\right)$, Stability $\left(f_{32}\right)$, Increase $\left(f_{33}\right)$ \\
\cline { 2 - 3 } & Market size & Increase $\left(f_{41}\right)$, Stability $\left(f_{42}\right)$, Decrease $\left(f_{43}\right)$ \\
\hline \multirow{2}{*}{ Environment } & Business environment & Stability $\left(f_{51}\right)$, Worse $\left(f_{52}\right)$, Better $\left(f_{53}\right)$ \\
\cline { 2 - 3 } & International relations & Stability $\left(f_{61}\right)$, Better $\left(f_{62}\right)$ \\
\hline
\end{tabular}

Hence, based on Eqn. (2), the most probable scenarios are shown in matrix $S$ : 


$$
S=\left(\begin{array}{llllllllll}
1 & 2 & 2 & 1 & 2 & 2 & 2 & 1 & 2 & 2 \\
1 & 2 & 1 & 2 & 1 & 1 & 2 & 1 & 2 & 1 \\
3 & 1 & 3 & 2 & 2 & 2 & 1 & 3 & 2 & 2 \\
1 & 2 & 1 & 3 & 2 & 2 & 1 & 1 & 2 & 2 \\
2 & 1 & 2 & 2 & 2 & 1 & 2 & 2 & 2 & 2 \\
2 & 1 & 2 & 2 & 1 & 2 & 1 & 2 & 1 & 2
\end{array}\right) .
$$

In step 3, the decision-maker specified favorability or non-favorability conditions of every option shown in the condition matrix $C$ :

$$
C=\left(\begin{array}{cccccccccc}
0 & 0 & 1 & 2 & 0 & 0 & 1 & 2 & 0 & 0 \\
1 & 2 & 2 & 1 & 1 & 2 & 2 & 1 & 1 & 2 \\
1,2 & 3 & 1 & 2,3 & 1 & 2,3 & 1 & 2,3 & 1,2 & 3 \\
1 & 2,3 & 1,2 & 3 & 1 & 2,3 & 1,2 & 3 & 1,2 & 3 \\
1,3 & 2 & 0 & 0 & 1,3 & 2 & 0 & 0 & 1,3 & 2 \\
0 & 0 & 2 & 1 & 2 & 1 & 2 & 1 & 2 & 1
\end{array}\right) .
$$

In step 4, applying FDANP, we obtained the final weights. In this order, the decision-maker specified the crisp direct influence matrix $A$ :

$$
A=\left(\begin{array}{llllll}
0 & 2 & 1 & 2 & 2 & 3 \\
3 & 0 & 3 & 4 & 2 & 1 \\
1 & 1 & 0 & 3 & 2 & 1 \\
1 & 2 & 4 & 0 & 3 & 1 \\
1 & 2 & 4 & 4 & 0 & 1 \\
4 & 4 & 3 & 3 & 2 & 0
\end{array}\right) .
$$

Consequently, according to Table 1 , we determined the fuzzy direct influence matrix $(\tilde{A})$ :

$$
\tilde{A}=\left(\begin{array}{cccccccccccccccccc}
f_{1}^{l} & f_{1}^{m} & f_{1}^{u} & f_{2}^{m} & f_{3}^{l} & f_{3}^{m} & f_{3}^{u} & & f_{4}^{m} & f_{5}^{l} & f_{5}^{m} & f_{5}^{u} & & f_{6}^{m} & \\
0 & 0 & .25 & .25 & .5 & .75 & 0 & .25 & .5 & .25 & .5 & .75 & .25 & .5 & .75 & .5 & .75 & 1 \\
.5 & .75 & 1 & 0 & 0 & .25 & .5 & .75 & 1 & .75 & 1 & 1 & .25 & .5 & .75 & 0 & .25 & .5 \\
0 & .25 & .5 & 0 & .25 & .5 & 0 & 0 & .25 & .5 & .75 & 1 & .25 & .5 & .75 & 0 & .25 & .5 \\
0 & .25 & .5 & .25 & .5 & .75 & .75 & 1 & 1 & 0 & 0 & .25 & .5 & .75 & 1 & 0 & .25 & .5 \\
0 & .25 & .5 & .25 & .5 & .75 & .75 & 1 & 1 & .75 & 1 & 1 & 0 & 0 & .25 & 0 & .25 & .5 \\
.75 & 1 & 1 & .75 & 1 & 1 & .5 & .75 & 1 & .5 & .75 & 1 & .25 & .5 & .75 & 0 & 0 & .25
\end{array}\right) .
$$

Now, applying Eqn. (4-10), we obtained the final weights of the criteria shown in Table 3.

Table 3. The criteria final weights $\left(W^{*}\right)$.

\begin{tabular}{|c|c|c|c|c|c|c|}
\hline Factor & Currency & Inflation & Competition & Market & environment & relations \\
\hline $\mathbf{W}^{*}$ & 0.15 & 0.16 & 0.19 & 0.20 & 0.17 & 0.13 \\
\hline
\end{tabular}


In step 5, by comparing the matrices $S$ and $C$, we can obtain matrix $Z$ showing the Robustness and Debility scores of different options in every scenario:

$$
B=\left(\begin{array}{rrrrrrrrrr}
-0.01 & 0.01 & -0.01 & -0.34 & -0.02 & 0.33 & 0.05 & -0.01 & -0.34 & -0.02 \\
0.13 & 0.27 & -0.17 & 0.05 & -0.43 & -0.17 & 0.27 & 0.13 & -0.11 & -0.17 \\
0.13 & -0.13 & 0.13 & -0.59 & -0.53 & 0.08 & -0.08 & 0.13 & -0.85 & -0.27 \\
0.13 & 0.27 & -0.17 & 0.05 & -0.43 & -0.17 & 0.27 & 0.13 & -0.11 & -0.17 \\
0.13 & 0.27 & 0.13 & -0.21 & 0.24 & 0.85 & -0.08 & 0.13 & -0.08 & 0.51
\end{array}\right) \text {, }
$$

Consequently, considering matrix $Z$ and applying Eqn. (11 and 12), we have robustness and debility of the options, vectors $R$ and $D$, as follows:

$$
\begin{aligned}
& R=\left[\begin{array}{lllll}
\frac{3}{10} & \frac{5}{10} & \frac{4}{10} & \frac{5}{10} & \frac{7}{10}
\end{array}\right]^{T}, \\
& D=\left[\begin{array}{llllll}
\frac{7}{10} & \frac{5}{10} & \frac{6}{10} & \frac{5}{10} & \frac{3}{10}
\end{array}\right]^{T} .
\end{aligned}
$$

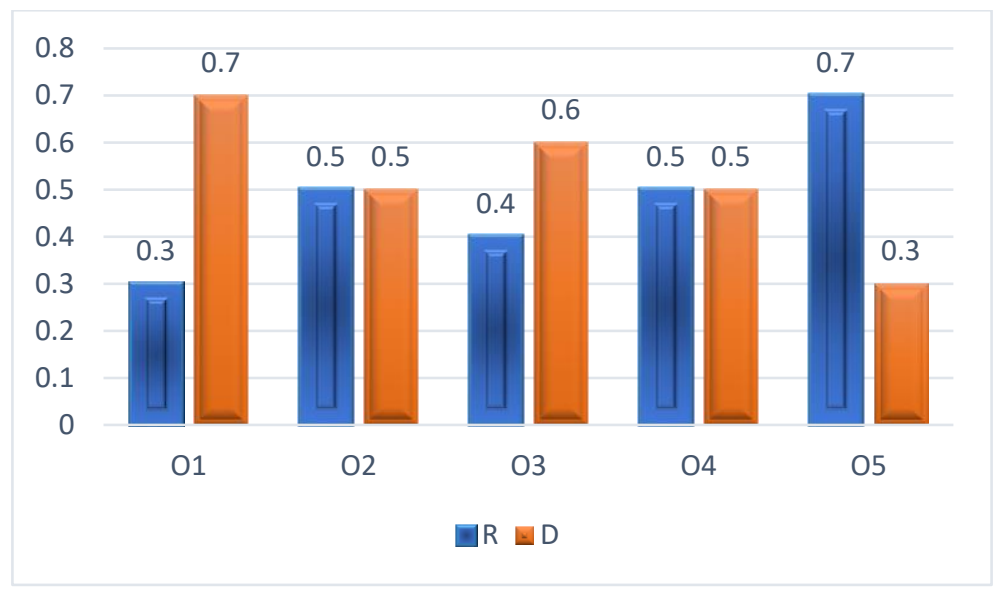

Figure 2. Robustness and debility of the options.

As shown in Figure 2, option 5, education service, is the only robust one. Finally, according to the relations (13 and 14), the robustness and debility of the main options levels are depicted in Figure 3. 




Figure 3. Robustness and debility of the product and service business.

Thus, as shown in Figure 3, the service business is more robust. To show our approach's sensibleness and effectiveness, we compare it with the MARA approach introduced by Sorourkhah et al. (2019). For example, consider $O_{1}$ as a single option the decision-maker has. The robustness and debility scores in 10 scenarios are shown in Table 4. (the first row is obtained according to the first row of matrix $Z$ in step 5 and the next row according to the MARA approach).

Table 4. Robustness and Debility of $O_{l}$ under MARA and the proposed approach.

\begin{tabular}{|c|c|c|c|c|c|c|c|c|c|c|c|c|}
\hline Approach & $\mathbf{1}$ & $\mathbf{2}$ & $\mathbf{3}$ & $\mathbf{4}$ & $\mathbf{5}$ & $\mathbf{6}$ & $\mathbf{7}$ & $\mathbf{8}$ & $\mathbf{9}$ & $\mathbf{1 0}$ & $\boldsymbol{R}$ & $\boldsymbol{D}$ \\
\hline $\boldsymbol{F D A N P}$ & - & + & - & - & - & + & + & - & - & - & $3 / 10$ & $7 / 10$ \\
\hline MARA & 0 & 0 & 0 & - & 0 & + & 0 & 0 & - & 0 & $1 / 10$ & $2 / 10$ \\
\hline
\end{tabular}

As shown in Table 4, by applying the proposed approach, we can determine the robustness or debility scores of $O_{1}$ in all possible scenarios. In contrast, we can evaluate it in only three scenarios by applying the MARA approach. It shows that our approach can present more acceptable results.

\section{Conclusions}

Every decision-maker makes many simple decisions affected by a few elements surrounded by a stable environment. This kind of decision does not need any extra analysis or formulation. As the environment becomes more complex, uncertain, and ambiguous, better decisions are drawn when an appropriate structure of the problem and evaluation of the multi-criteria nature of the problem is explicitly established (Ocampo et al., 2019). During the last years, a growing number of studies have investigated complexity (Kim et al., 2021). Complexity increases with the number of elements (criteria, scenarios, options, alternatives, etc.) and interdependencies (Tadić et al., 2014). MCDM can be helpful when we encounter a situation in which complexity increases (Hu et al., 2014; Arjomandi et al., 2021; Kavta and Goswami, 2021). Nevertheless, because this approach is not suitable for the fuzzy environment of the real world (Hu et al., 2018), so many researchers addressed incorporating the fuzzy set theory in ANP, DEMATEL, or both to deal with uncertainty and complexity (Ocampo et al., 2020). But, as mentioned before, these very approaches do not consider the most critical aspect of uncertainty, future uncertainty (Sorourkhah et al., 2019). 
Scenario Planning (SP), Strategic Choice Approach (SCA), and Robustness Analysis (RA) are three different approaches that consider future uncertainty. Having some distinct advantages, none of them can deal with both aspects of complexity. Scenario planning (SP) often includes only three, four, or five scenarios; therefore, it can not deal with various criteria affecting the problem to address the first aspect of the complexity, numerous variables affecting the consequences of the decision. The strategic choice approach is reliable for handling uncertainty. However, it cannot model the relationships among the criteria and their possible future situations. And robustness analysis, which is able to handle one aspect of the complexity, the number of the criteria, scenarios, etc., does not consider the criteria interdependencies.

The real-world situation, comprising increasing criteria and alternatives; the criteria interdependency; environmental changes affecting the structure of the organization; the vagueness of the verbal judgments; and increasing uncertainty about possible futures, forces the decisionmakers to consider these two critical elements, complexity and uncertainty, in their decisionmaking approach (Sorourkhah and Edalatpanah, 2021). In this regard, we applied a combination of MARA and FDANP, which can consider numerous scenarios, criteria, and alternatives as well as the interdependency among criteria to address the complexity aspect, and the environmental uncertainty by examining the option performance among the different possible futures as well as the fuzzy nature of the problem owner's judgments to address the uncertainty aspect. Another advantage of our approach is that it is not time-consuming at all. Using the matrix approach allows us to receive the judgments of the decision-makers quickly and, unlike the classic robustness analysis approach, determine the situation of the whole decisions in all the future scenarios. Applying this method shows that option 5, education service, is the most robust. As education is vital to individuals for their personal and professional accomplishments (Baykasoğlu et al., 2013) and its need is constantly increasing (Su et al., 2016), this option seems reasonable.

Finally, it should be said that some limitations of this study require further examination: for example, as the future scenarios are determined by decision-makers judgmentally, using a structured, scientific method, like artificial intelligence, can be helpful; As fuzzy set theory needs auxiliary information and pre-assumptions, like membership functions, to describe the degree of imprecision (Song et al., 2020), applying more acceptable approaches, like q-rung orthopair fuzzy Einstein model (Akram et al., 2021), can be helpful as well. And because in a decision-making process, uncertain factors influence decision-makers, we can apply the new entropy measure for Pythagorean Fuzzy Set (PFS) introduced by Wang et al. to determine attribute weights (Wang et al., 2021). Nevertheless, we believe that the most severe weakness of the proposed approach is that it cannot analyze quantitative data, precisely the financial results of each decision. In our future study, we are going to address this problem.

\section{Conflict of Interest}

The authors confirm that there is no conflict of interest to declare for this publication.

\section{Acknowledgement}

The authors would like to thank the referees for their critical comments and valuable suggestions which helped the authors to improve the presentation of this paper. 


\section{References}

Akram, M., Naz, S., Edalatpanah, S.A., \& Mehreen, R. (2021). Group decision-making framework under linguistic q-rung orthopair fuzzy Einstein models. Soft Computing, 25(15), 10309-10334.

Arjomandi, M.A., Dinmohammadi, F., Mosallanezhad, B., \& Shafiee, M. (2021). A fuzzy DEMATEL-ANPVIKOR analytical model for maintenance strategy selection of safety critical assets. Advances in Mechanical Engineering, 13(4), 1-21.

Avikal, S., Singhal, R., Sajwan, R., Tiwari, R., \& Singh, R. (2020). Selection of best power supply source for telecom towers in remote areas. International Journal of Mathematical, Engineering and Management Sciences, 5(5), 913-925.

Azar, A., \& Sorourkhah, A. (2015). Designing a model for three-dimensional robustness analysis: A case study of Iran Khodro machine tools industries company. Indian Journal of Science and Technology, $8(28), 1-5$.

Baykasoğlu, A., Kaplanoğlu, V., Durmuşoğlu, Z.D.U., \& Şahin, C. (2013). Integrating fuzzy DEMATEL and fuzzy hierarchical TOPSIS methods for truck selection. Expert Systems with Applications, 40(3), 899-907. https://doi.org/10.1016/j.eswa.2012.05.046.

Busyra, S.H.I., \& Ardi, R. (2020). Risk analysis of upstream halal supply chain in meat industry in Indonesia using DEMATEL-based ANP. In 2020 IEEE International Conference on Industrial Engineering and Engineering Management (pp. 706-710). IEEE. Singapore, Singapore.

Chang, B., Chang, C.W., \& Wu, C.H. (2011). Fuzzy DEMATEL method for developing supplier selection criteria. Expert Systems with Applications, 38(3), 1850-1858.

Chen, M.F., Tzeng, G.H., \& Ding, C.G. (2008). Combining fuzzy AHP with MDS in identifying the preference similarity of alternatives. Applied Soft Computing, 8(1), 110-117. doi: https://doi.org/10.1016/j.asoc.2006.11.007.

Chutia, R., \& Gogoi, M.K. (2018). Fuzzy risk analysis in poultry farming based on a novel similarity measure of fuzzy numbers. Applied Soft Computing, 66, 60-76.

Dinçer, H., Yüksel, S., \& Martínez, L. (2019). Interval type 2-based hybrid fuzzy evaluation of financial services in E7 economies with DEMATEL-ANP and MOORA methods. Applied Soft Computing, 79, 186-202.

Edalatpanah, S.A. (2020). Neutrosophic structured element. Expert Systems, 37(5), 1-17.

Greiner, R., Puig, J., Huchery, C., Collier, N., \& Garnett, S.T. (2014). Scenario modelling to support industry strategic planning and decision making. Environmental Modelling \& Software, 55, 120-131.

Hamedi, H., \& Mehdiabadi, A. (2020). Entrepreneurship resilience and Iranian organizations: Application of the fuzzy DANP technique. Asia Pacific Journal of Innovation and Entrepreneurship, 14(3), 231-247.

Hsu, C.H., Wang, F.K., \& Tzeng, G.H. (2012). The best vendor selection for conducting the recycled material based on a hybrid MCDM model combining DANP with VIKOR. Resources, Conservation and Recycling, 66, 95-111.

Hu, S.K., Liou, J., Chuang, Y.C., \& Tzeng, G.H. (2018). New hybrid FMADM model for mobile commerce improvement. Technological and Economic Development of Economy, 24(5), 1801-1828.

Hu, S.K., Lu, M.T., \& Tzeng, G.H. (2014). Exploring smart phone improvements based on a hybrid MCDM model. Expert Systems with Applications, 41(9), 4401-4413.

Huang, C.Y. (2012, Nov). The non-additive Choquet integration in the fuzzy DNP framework for evaluating co-dependent technology transfer models. In 2012 International conference on Fuzzy Theory and Its Applications (pp. 125-130). IEEE. Taichung, Taiwan. 
Huang, C.Y., \& Ting, Y.H. (2012, Nov). Derivations of factors influencing the word-of-mouth marketing strategies for smart phone applications by using the fuzzy DEMATEL based network process. In 2012 International conference on Fuzzy Theory and Its Applications (pp. 42-47). IEEE. Taichung, Taiwan.

Karuppiah, K., Sankaranarayanan, B., \& Ali, S.M. (2020). A fuzzy ANP-DEMATEL model on faulty behavior risks: implications for improving safety in the workplace. International Journal of Occupational Safety and Ergonomics (accepted). doi:https://doi.org/10.1080/10803548.2020.1847486.

Kavta, K., \& Goswami, A.K. (2021). A methodological framework for a priori selection of travel demand management package using fuzzy MCDM methods. Transportation, 48(6), 3059-3084.

Kharisma, S.A., \& Ardi, R. (2020, Dec). Supply chain risk asssessment of generic medicine in Indonesia using DEMATEL-based ANP (DANP). In 2020 IEEE International Conference on Industrial Engineering and Engineering Management (pp. 716-720). IEEE. Singapore, Singapore.

Kim, S.Y., Nguyen, M.V., \& Dao, T.T.N. (2021). Prioritizing complexity using fuzzy DANP: case study of international development projects. Engineering, Construction and Architectural Management, 28(4), 1114-1133. doi:https://doi.org/10.1108/ECAM-04-2020-0265.

Lin, C.J., \& Wu, W.W. (2008). A causal analytical method for group decision-making under fuzzy environment. Expert Systems with Applications, 34(1), 205-213.

Lin, T., \& Pham, H. (2019). Reliability and cost-benefit analysis for two-stage intervened decision-making systems with interdependent decision units. International Journal of Mathematical, Engineering and Management Sciences, 4(3), 531-541. doi:https://dx.doi.org/10.33889/IJMEMS.2019.4.3-043.

Liou, J.J.H., Hsu, C.C., Li, C.S.J., Pineda, P.J.G., \& Chang, G.W. (2018). Developing a successful aerotropolis by using a hybrid model under information uncertainty. Technological and Economic Development of Economy, 24(3), 1080-1103. doi:https://doi.org/10.3846/20294913.2017.1289484.

Månsson, A. (2016). Energy security in a decarbonised transport sector: A scenario based analysis of Sweden's transport strategies. Energy Strategy Reviews, 13-14, 236-247.

Nakhjavani, A., Mollaverdi, N., \& Rafiee, F.M. (2014). Hybrid approach of ANP and DEMATEL for selecting optimal maintenance strategy: a case study in the pharmaceutical industry. Journal of Applied Research on Industrial Engineering, 1(4), 230-249.

Namen, A.A., Bornstein, C.T., \& Rosenhead, J. (2010). The use of robustness analysis for planning actions in a poor Brazilian community. Pesquisa Operacional, 30(2), 267-280.

Ocampo, L.A., Clark, E.E., Tanudtanud, K.V.G., Ocampo, C.O.V., Impas, S.C.G., \& Vergara, V.G., Pastoril, J., Tordillo, J.A.S. (2015). An integrated sustainable manufacturing strategy framework using fuzzy analytic network process. Advances in Production Engineering \& Management, 10(3), 125-139.

Ocampo, L., Deiparine, C.B., \& Go, A.L. (2020). Mapping strategy to best practices for sustainable food manufacturing using fuzzy DEMATEL-ANP-TOPSIS. Engineering Management Journal, 32(2), 130150. doi:https://doi.org/10.1080/10429247.2020.1733379.

Ocampo, L.A., Himang, C.M., Kumar, A., \& Brezocnik, M. (2019). A novel multiple criteria decisionmaking approach based on fuzzy DEMATEL, fuzzy ANP and fuzzy AHP for mapping collection and distribution centers in reverse logistics. Advances in Production Engineering \& Management, 14(3), 297-322.

Rosenhead, J. (1980). Planning under uncertainty: II. A methodology for robustness analysis. Journal of the Operational Research Society, 31(4), 331-341.

Rosenhead, J. (2011). Robustness analysis. In Cochran, J.J., Cox, Jr. L.A., Keskinocak, P., Kharoufeh, J.P., \& Smith, J.C. (eds.) Wiley Encyclopedia of Operations Research and Management Science: John Wiley \& Sons. https://doi.org/10.1002/9780470400531.eorms0976. 
Rudd, J.M., Greenley, G.E., Beatson, A.T., \& Lings, I.N. (2008). Strategic planning and performance: Extending the debate. Journal of Business Research, 61(2), 99-108.

Sagnak, M., Ada, E., Kazancoglu, Y., \& Mishra, A. (2020). Integration of lean approach with energy efficiency: Application in kitchenware manufacturing company. International Journal of Mathematical, Engineering and Management Sciences, 5(6), 1128-1139.

Seker, S., \& Zavadskas, E.K. (2017). Application of fuzzy DEMATEL method for analyzing occupational risks on construction sites. Sustainability, 9(11), 1-19. doi:https://doi.org/doi:10.3390/su9112083.

Salimi, P.S., \& Edalatpanah, S.A. (2020). Supplier selection using fuzzy AHP method and D-numbers. Journal of Fuzzy Extension and Applications, 1(1), 1-14.

Song, W., Zhu, Y., \& Zhao, Q. (2020). Analyzing barriers for adopting sustainable online consumption: A rough hierarchical DEMATEL method. Computers \& Industrial Engineering, 140, 106279.

Sorourkhah, A., Kafaki, S.B., Azar, A., \& Nikabadi, M.S. (2018). Matrix approach to robustness analysis for strategy selection. International Journal of Industrial Mathematics, 10(3), 261-269.

Sorourkhah, A., Kafaki, S.B., Azar, A., \& Nikabadi, M.S. (2019). A fuzzy-weighted approach to the problem of selecting the right strategy using the robustness analysis (Case study: Iran Automotive Industry). Fuzzy Information and Engineering, 11(1), 39-53.

Sorourkhah, A., \& Edalatpanah, S.A. (2021). Considering the criteria interdependency in the matrix approach to robustness analysis with applying fuzzy ANP. Fuzzy Optimization and Modeling Journal, 3(2), 2233.

Su, C.H., Tzeng, G.H., \& Hu, S.K. (2016). Cloud e-learning service strategies for improving e-learning innovation performance in a fuzzy environment by using a new hybrid fuzzy multiple attribute decisionmaking model. Interactive Learning Environments, 24(8), 1812-1835.

Sudarmin, A.C., \& Ardi, R. (2020, Dec). DEMATEL-based analytic network process (ANP) approach to assess the vaccine supply chain risk in Indonesia. In 2020 IEEE International Conference on Industrial Engineering and Engineering Management (pp. 726-730). IEEE. Singapore, Singapore.

Taati, S.N., \& Dooki, A.E. (2017). A hybrid method of Fuzzy DEMATEL/AHP/VIKOR approach to rank and select the best hospital nurses of a year: A case study. Journal of Applied Research on Industrial Engineering, 4(2), 116-132. doi:https://doi.org/10.22105/jarie.2017.95312.1017.

Tadić, S., Zečević, S., \& Krstić, M. (2014). A novel hybrid MCDM model based on fuzzy DEMATEL, fuzzy ANP and fuzzy VIKOR for city logistics concept selection. Expert Systems with Applications, 41(18), 8112-8128. doi:https://doi.org/10.1016/j.eswa.2014.07.021.

Vinodh, S., \& Swarnakar, V. (2015). Lean six sigma project selection using hybrid approach based on fuzzy DEMATEL-ANP-TOPSIS. International Journal of Lean Six Sigma, 6(4), 313-338.

Wang, L., Garg, H., \& Li, N. (2021). Pythagorean fuzzy interactive Hamacher power aggregation operators for assessment of express service quality with entropy weight. Soft Computing, 25(2), 973-993.

Wu, W.W., \& Lee, Y.T. (2007). Developing global managers' competencies using the fuzzy DEMATEL method. Expert Systems with Applications, 32(2), 499-507. 